\title{
Asymmetric Resistive Switching Dynamics in BaTiO3 Tunnel Junctions
}

Mengdi Qian, Ignasi Fina, Florencio Sánchez and Josep Fontcuberta

M. Qian, Dr. I. Fina,* Dr. F. Sánchez, and Prof. J. Fontcuberta*

Institut de Ciència de Materials de Barcelona (ICMAB-CSIC), Campus UAB, Bellaterra 08193, Catalonia, Spain

E-mail:ifina@icmab.es; fontcuberta@icmab.cat

Keywords: ferroelectric tunnel junctions, ferroelectric dynamics, $\mathrm{BaTiO}_{3}$ films, memristor, neuromorphic computing

The resistive switching associated to polarization reversal observed in $\mathrm{BaTiO}_{3}$ tunnel junctions, is studied in detail with focus on the dynamics of the ferroelectric domain switching. It is observed that the transition between the high-resistive state (HRS) and the low-resistive state (LRS) is largely asymmetric being smooth from LRS-to-HRS but proceed via avalanches in the HRS-to-LRS transitions. We show that this distinct behavior is related to the presence of imprint field in the junction and has important consequences on the junction's performance. 


\section{Introduction}

Ferroelectric tunnel junctions in capacitor metal/ferroelectric/metal structures are receiving enormous interest for their potential use as high density (scalability), low energy consumption (small writing/reading voltage) and fast operation non-volatile random access memories (RAM $)^{[1-3]}$. Indeed, switching of the ferroelectric polarization by a suitable writing voltage leads to a changes of the junction resistance with a large resistance contrast between the high resistance state (HRS) and the low resistance state (LRS) than can reach $10^{4}-10^{8} \%{ }^{[4-17]}$ Therefore hysteric I-V characteristics with resistance jumps at the coercive voltages are observed (sketched in Figure 1a). These binary easily controllable states are of interest for data storage and digital computing. For that reason ferroelectric RAM (FeRAM) are being intensively investigated. ${ }^{[18]}$ The requirements of growth of single crystalline-like epitaxial oxide layers, with a low defect density to allow charge-tunneling transport channel, are extremely demanding but progress in oxide-based thin film growth technologies have permitted to have tunnel FeRAM at reach. ${ }^{[1]}$

The extreme thin structure of the dielectric barrier, on the other hand, implies that the ferroelectric domains, whose size decreases with film thickness, must have also a lateral size comparable to the film thickness, that is: few nanometers. Several important consequences emerge from this size scaling. First is that, if ferroelectric domains under the electrode can switch independently under suitable voltage bias, then multiple HRS and LRS can be achieved in a given capacitor and, as polarization switching is known to be dependent on the amplitude and duration of the polarization voltages,${ }^{[19-22]}$ memristive response can be achieved. ${ }^{[17]}$ Second, ferroelectric/electrode electrostatic and microstructural effects should play a major role on the time-dependent dynamics of the polarization switching. ${ }^{[23-25]}$ Most commonly, asymmetric electrodes are used in ferroelectric capacitors and as the nature of the corresponding metal/ferroelectric and ferroelectric/metal interfaces are different. Thus, asymmetric polarization switching for positive/negative bias can occur, and it has been experimentally observed. ${ }^{[22,26-30]}$ When refereeing to the resistive switching controlled by ferroelectric polarization, the same arguments given above suggest that the HRS-to-LRS and LRS- 
to-HRS processes could be also different, implying an asymmetric response when reversing the writing electric fields. Understanding and controlling these effects is pivotal to exploit the potential multistate of the ferroelectric memory. Even more, deep knowledge on asymmetric dynamics is crucial for ferroelectric tunnel junctions implementation in neuromorphic circuits, where timedependent action potentials trigger the synapse responses. ${ }^{[31]}$

In the particular case of ferroelectric tunnel barriers, Chanthboula et al. ${ }^{[17]}$ pioneeringly reported the impact of the polarization switching dynamics on the measured change of resistance (so called “electroresistance, ER, or tunnel electroresistance, TER"). These authors observed that under a given voltage bias larger than the coercive field (at a given measuring frequency) the switching from LRSto HRS was gradual, expanding along a range of voltages. The smooth transition from LRS to HRS was successfully modeled by assuming the contributions of domain nucleation and expansion mechanisms on the domain dynamics. In contrast, the reverse process (HRS-to-LRS) was observed to be more abrupt. This observation, reminiscent of the asymmetric polarization reversal in ferroelectric capacitors ${ }^{[22,26,28-30]}$ may imply a different responsivity of LRS and HRS to external stimuli, thus impacting the memristive response depending on the sign of the biasing electric field.

Here we focus on the comparison of the resistive transition from LRS-to-HRS and HRS-to LRS in $\mathrm{BaTiO}_{3}$ ferroelectric tunnel junctions, triggered by electric fields of different polarity. We show that the dynamics of polarization and the concomitant resistive switching of the $\mathrm{BaTiO}_{3}$ junctions are radically different for both polarizations. The resistive transition from LRS-to-HRS is smooth, suggesting it is dominated by random nucleation and growth of polar domains. In contrast, the HRSto-LRS display avalanche-like features which depend on the amplitude and frequency of the applied electric field. We show that these asymmetric resistive transitions are intimately related to the presence of imprint fields in the device and we argue that its understanding is crucial for applications, such as neuromorphic computing, where the dynamics of the resistive transition is pivotal. 
$\mathbf{a}$

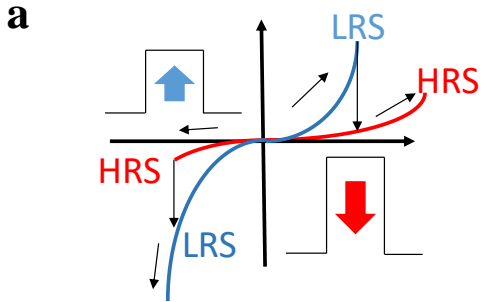

c

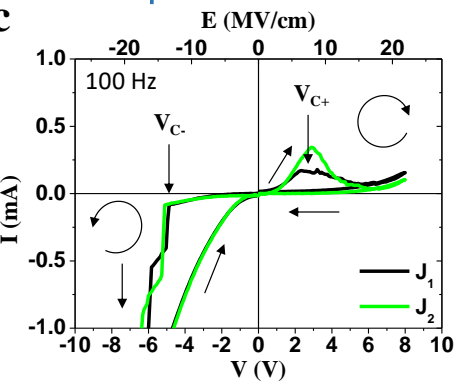

b

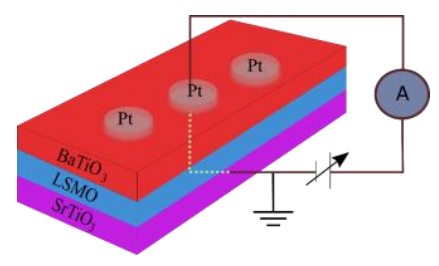

d

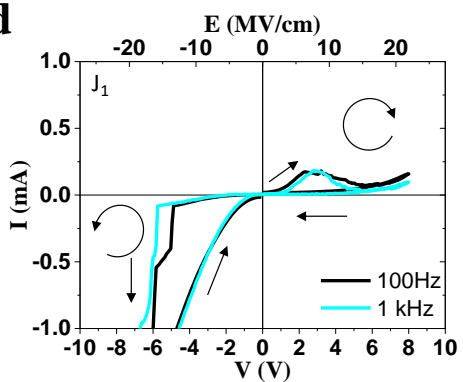

Figure 1. (a) Sketch of the switching process from the low resistance state (LRS) to the high resistance state (HRS) in the I-V characteristics of a ferroelectric tunnel junction, when the barrier height changes from LRS to HRS when reversing polarization (up/down color arrows) as indicated (b) Sketch of the Pt/BTO/LSMO junction and electrical measurement configuration. (c) I-V loops following the $-8 \mathrm{~V}$ to $+8 \mathrm{~V}$ to $-8 \mathrm{~V}$ path of two different junctions ( $\mathrm{J}_{1}$ (black), $\mathrm{J}_{2}$ (green)), measured at $100 \mathrm{~Hz}$. (d) I-V loops of the $\mathrm{J}_{1}$ junction measured at $100 \mathrm{~Hz}$ (black) and $1 \mathrm{kHz}$ (cyan).

\section{Results}

In Figure 1c, we show illustrative $\mathrm{I}-\mathrm{V}$ loops (junctions $\mathrm{J}_{1}$ and $\mathrm{J}_{2}$ ) recorded in the $\pm 8 \mathrm{~V}$ range at 100 Hz. Data indicate that I-V loops have a clockwise and anticlockwise hysterical behavior for V $>0$ and $\mathrm{V}<0$ regions, respectively. In the $\mathrm{V}>0$ region (positive voltage applied to the top Pt electrode), the clockwise rotation of I-V implies a change from low-resistance state (LRS) to a high-resistance state (HRS) upon increasing $\mathrm{V}$, occurring at a critical voltage $\left(\mathrm{V}_{\mathrm{C}+}\right) \mathrm{V}_{\mathrm{C}+} \approx 3 \mathrm{~V}$. The junction remains in the HRS when retreating voltage until a critical voltage $\left(\mathrm{V}_{\mathrm{C}-}\right) \mathrm{V}_{\mathrm{C}-} \approx-5 \mathrm{~V}$, where the LRS is recovered (anticlockwise rotation). When reducing the amplitude of the $\mathrm{V}<0$ field, the LRS is preserved. The HRS is only restored again if $\mathrm{V}_{\mathrm{C}+}$ is overpassed again. It is obvious in Figure 1c that the resistive switching signatures are radically different depending on the sense of the switching (HRS-to-LRS and viceversa). It is of limited amplitude and smooth from LRS to HRS, whereas it of large amplitude and displays several steps during the HRS to LRS transition. This different behavior is observed in most of the junctions fabricated on the sample. Data for other junctions are included in Figure S1. In Figure 1d, we show the I-V loops of $\mathrm{J}_{1}$ recorded at different frequencies ( $100 \mathrm{~Hz}$ and 1 $\mathrm{kHz}$ ). Inspection of these data reveals two prominent features. First, in the $\mathrm{V}>0$ region the switching 
from LRS to HRS appears at higher $\mathrm{V}$ and the width and amplitude varies with the measuring frequency. Second, in the $\mathrm{V}<0$ region, the characteristics steps in I-V curve signaling the transition from HRS to LRS also occur at higher voltages when increasing frequency and the amount and height of the steps in $\mathrm{I}(\mathrm{V})$ are also modified. This is a common trend observed in most junctions as seen in Figures S2 and S3 in Supplementary Information. In the following, we analyze in detail these observations.

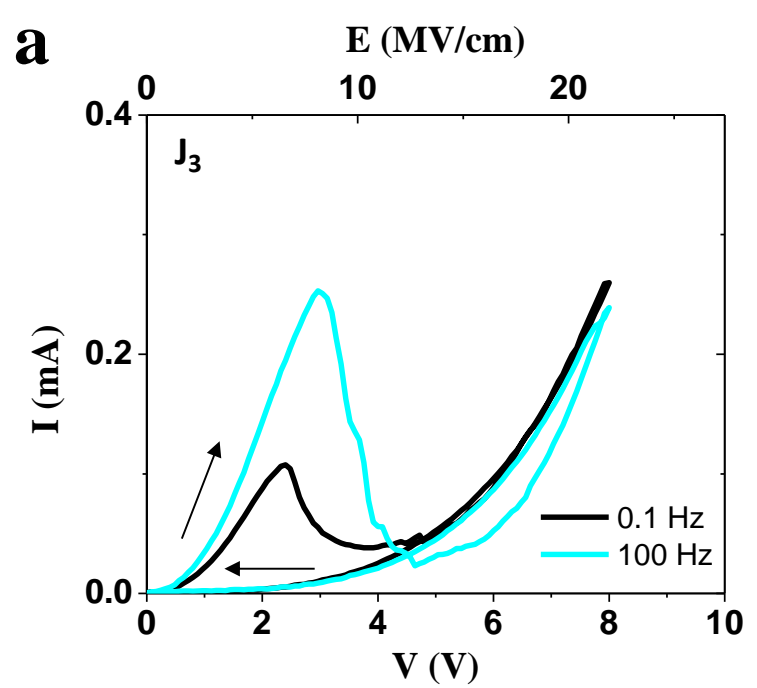

b

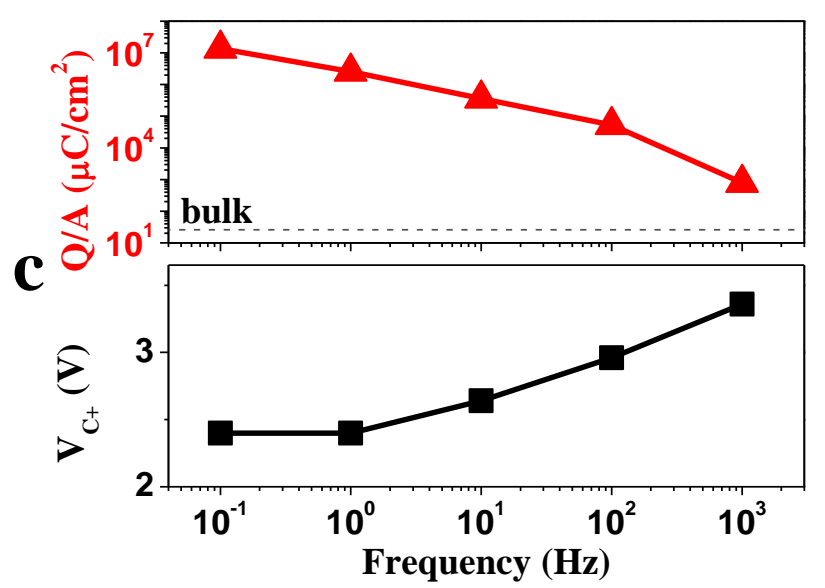

Figure 2. (a) Positive $\mathrm{V}>0$ branch of the $\mathrm{I}-\mathrm{V}$ loops of junction $\mathrm{J}_{3}$ recorded using triangular pulses of frequencies $0.1 \mathrm{~Hz}$ and $100 \mathrm{~Hz}$. (b) Integrated area under the $\mathrm{I}(\mathrm{t})$ current peak $(\mathrm{Q})$, normalized by the junction area $(\mathrm{Q} / \mathrm{A})$, as a function on the measuring frequency. (c) Dependence of the position of the $\mathrm{I}-\mathrm{V}$ maxima $\left(\mathrm{V}_{\mathrm{C}+}\right)$ on the measuring frequency.

In Figure 2a, we show a zoom of the $\mathrm{V}>0$ region of the $\mathrm{I}-\mathrm{V}$ loops of junction $\mathrm{J}_{3}$ recorded using different frequencies $(0.1$ and $100 \mathrm{~Hz})$. Similar data for other junctions are included in Figure S2. In Figure 2a, the trends already identified in Figure 1c, namely the shift of the voltage where switching occurs $\left(\mathrm{V}_{\mathrm{C}+}\right)$ and the change of the corresponding peak (increase of amplitude) for increasing measurement frequency can be more clearly appreciated because the selected measurement frequencies shown in Figure $2 \mathrm{a}$ differ by 3 orders of magnitude. In Figure 2b, we show the timeintegrated area under the switching current peak $\left(Q=\int I(t) d t\right)$ divided by the contact area (A) as a function of frequency. Q/A is a measure of the specific charge that has flowed across the measuring device during the transition from LRS to HRS. It can be observed that $\mathrm{Q} / \mathrm{A}$ is as large as $10^{7} \mu \mathrm{C} / \mathrm{cm}^{2}$ at the smallest frequency $(0.1 \mathrm{~Hz})$ and decreases rapidly upon increasing frequency. Irrespective of 
the measurement frequency the Q/A values are orders of magnitude larger than the polarization of bulk BTO $\left(\approx 26 \mu \mathrm{C} / \mathrm{cm}^{2}\right.$, dashed line in Figure $\left.2 \mathrm{~b}\right)$ implying that the measured current is dominated by transport current in the device rather than by the displacive current consequence of the ferroelectric polarization switching. Therefore, it can be safely concluded that the observed switching peak at $\mathrm{V}_{\mathrm{C}+}$ indicates an actual change of resistance from LRS to HRS when increasing V rather than the polarization switching displacive current. In Figure 2c, we summarize the frequency-dependence of the $\mathrm{V}_{\mathrm{C}+}$ peak. It is clear that $\mathrm{V}_{\mathrm{C}+}$ increases with frequency. The roughly linear dependence of $\mathrm{V}_{\mathrm{C}+}$ on $\log (v)$ (Figure 2c) is a common frequency dependence of the coercive fields in ferroelectrics. ${ }^{[32]}$ Therefore, we argue that resistive switching maxima occur at the ferroelectric coercive voltage, and we assume that the switching peak correspond to the switching from LRS to HRS controlled by the polarization reversal of BTO.

In ferroelectric devices backswitching can be a relevant contribution to the polarization dynamics. ${ }^{[30,33]}$ To address this point, we have written a $\mathrm{P}^{\uparrow}$ state (where $\mathrm{P}^{\uparrow}$ denotes polarization pointing towards $\mathrm{Pt})$ by applying a suitable negative pulse $\left(\tau_{w}=1 \mathrm{~s}\right)$ equivalent to a measuring frequency of $0.5 \mathrm{~Hz}$, and subsequently we have recorded $\mathrm{I}-\mathrm{V}$ loops $(\mathrm{V}>0)$ with a delay times $\left(\tau_{D}\right)$ between writing and reading voltage pulses as indicated in Figure 3 (sketches). We first recorded an I-V curve with $\tau_{D}=0$ (green curve in Figure 3). Next we have rewritten the same initial state and measured the I-V loop with $\tau_{D}=1 \mathrm{~s}$ (red curve in Figure 3). We notice in Figure 3 that when a delay time $\tau_{D}$ is introduced between writing and reading pulses, the amplitude of the switching peak, as thus the LRS to HRS transition is reduced, but the resistance on the HRS is virtually identical and the retreat curves coincide. This observation indicates that during the delay time $\tau_{D}$ (writing voltage zeroed) some polar domains have spontaneously switched back from $\mathrm{P}^{\uparrow}$ to $\mathrm{P}^{\downarrow}$. Therefore, an imprint Eimp field exists, governing the backswitching of polarization and it is directed from Pt to LSMO as indicated in Figure 3. 


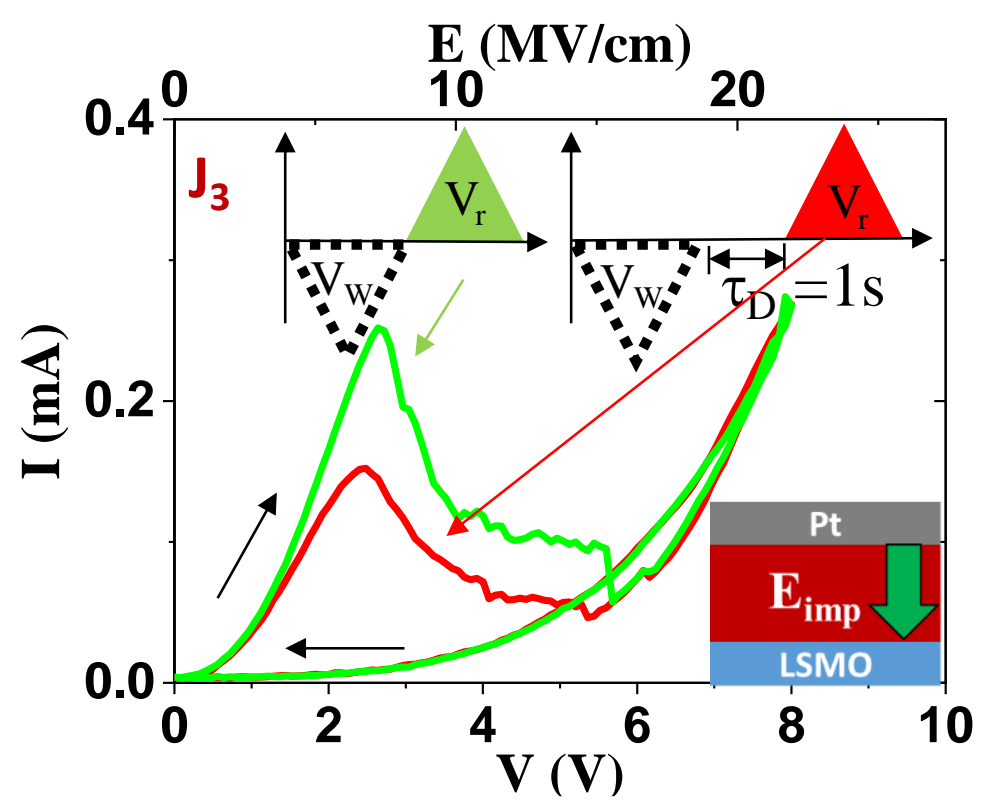

Figure 3. I-V reading pulses recorded after prepolarizating (polarization up, $\mathrm{P}^{\uparrow}$ ) the junction (writing) with a $\mathrm{V}=-8 \mathrm{~V}$ pulse of $\tau_{w}=1 \mathrm{~s}$, with a delay time $\tau_{D}$ of $\tau_{D}=0$ (green curve) and $\tau_{D}=1 \mathrm{~s}$ (red curve). Top sketches indicate the sequence of prepoling writing (dashed triangle) and reading voltages $V(t)$ pulses (green and read triangles). Bottom sketch indicates the direction (green arrow) of the imprint electric field ( $\mathrm{E}_{\mathrm{imp}}$ ) (from Pt to LSMO) responsible for backswitching of $\mathrm{P}$.

We turn now to the HRS to LRS switching occurring at $\mathrm{V}<0$. We have collected I-V curves in the $\mathrm{V}<0$ range (after the initial $\mathrm{V}=+8 \mathrm{~V}$ and $\mathrm{V}=0$ with $\tau_{W}=1 \mathrm{~s}$ ). We have recorded $\mathrm{I}-\mathrm{V}$ loops when varying the frequency of the $\mathrm{V}(\mathrm{t})$ pulse. In Figure $4(\mathrm{a}-\mathrm{d})$, we show data of $\mathrm{J}_{4}$ junction at some illustrative frequencies $(1,10,100,1000 \mathrm{~Hz})$. Similar data for other junctions are included in Figure S3. Inspection of the I-V curve recorded at $1 \mathrm{~Hz}$ (Figure 4a) shows that when increasing the negative bias, well defined steps appear at $\mathrm{V}_{\mathrm{C}-, 1}=-4.5 \mathrm{~V}$ and $\mathrm{V}_{\mathrm{C}-2}=-6.2 \mathrm{~V}$, respectively which indicate partial transitions from HRS to LRS. When increasing the measuring frequency, the first step in I-V occurs at somewhat larger (negative) voltage, for instance $\mathrm{V}_{\mathrm{C}_{-}}=-5.5 \mathrm{~V}$ at $10 \mathrm{~Hz}$ (Figure $4 \mathrm{~b}$ ). In Figure $4 \mathrm{e}$ we show the frequency-dependent of $\mathrm{V}_{\mathrm{c}-, 1}$ values for three different junctions. As indicated above, a gradual shift of $\mathrm{V}_{\mathrm{c}-, 1}$ to larger negative voltage is consistently observed for the different measured junctions. 

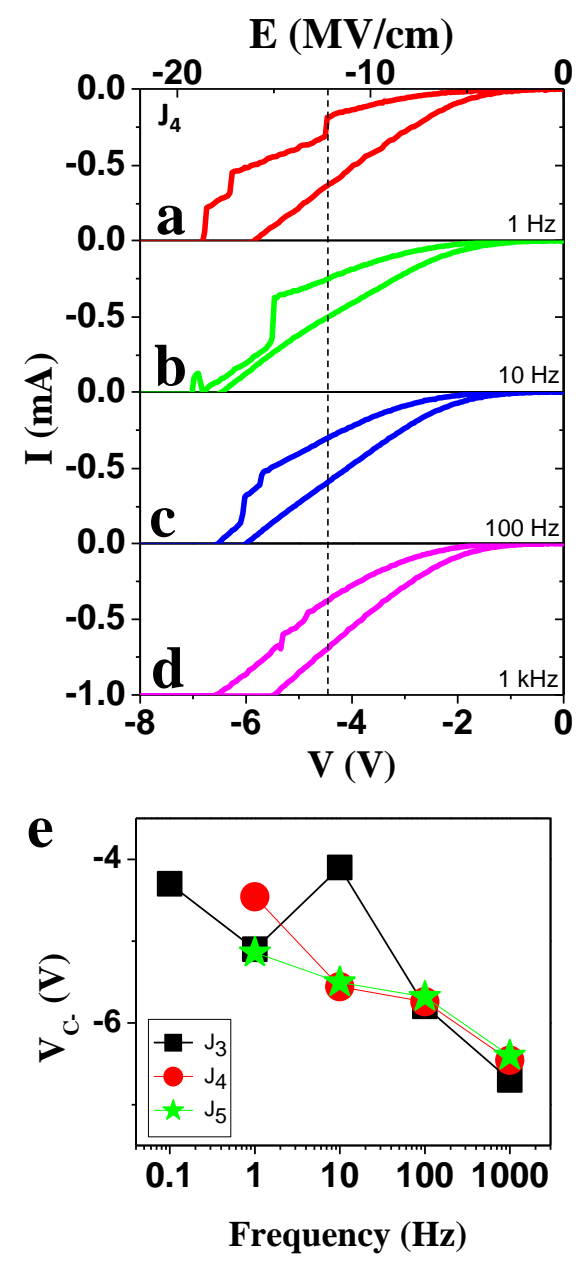

Figure 4. (a-d) Dependence of the HRS to LRS transitions in the reverse side $\left(\mathrm{V}<0\right.$ ) (junction $\mathrm{J}_{4}$ ) on the measuring (reading) frequency. Prior to measurement the junctions have been pre-poled with a $\mathrm{V}=+8 \mathrm{~V}$ triangular pulse of $\tau_{W}=1 \mathrm{~s}$. Dashed vertical line indicates the position of the $1^{\text {st }}$ current step occurring at $\mathrm{V}_{\mathrm{C}-1,1}=-4.5 \mathrm{~V}(1 \mathrm{~Hz})$. (e) First step current onset $\left(\mathrm{V}_{\mathrm{C}-}\right)$ of $\mathrm{J}_{3}$ (black squares), $\mathrm{J}_{4}$ (red circles) and $\mathrm{J}_{5}$ (green stars) junctions versus frequency.

The I-V trends in Figure 4 are reminiscent of domain avalanches promoted by the $V(t)$ excitation in ferroelastic $^{[34,35]}$, ferroelectrics ${ }^{[26,36,37]}$, and other materials ${ }^{[38]}$. To get additional insight, we recorded $\mathrm{I}-\mathrm{V}$ curves at $\mathrm{V}<0$ as above, and compared with the one obtained after returning to $\mathrm{V}=0$ and, without re-poling the sample (that is without applying any $\mathrm{V}>0$ pulse), increase again the negative voltage after a delay time $\tau_{D}=0$ or $1 \mathrm{~s}$. Measuring cycles are indicated in the sketches in Figures 5a and $5 \mathrm{~b}$, where we also show the results obtained for $\tau_{D}=0$ (junction $\mathrm{J}_{5}$ ) and $\tau_{D}=1 \mathrm{~s}$ (junction $\mathrm{J}_{6}$ ), respectively. In Figure 5a, it can be appreciated that the initial I-V loop (green curve), recorded at 10 $\mathrm{Hz}$, in agreement with data in Figure 1 and Figure 4, clearly displays conductance steps. Within the proposed framework, the jumps in I-V mean that polar $\mathrm{P}^{\uparrow}$ domains have been switched to $\mathrm{P}^{\downarrow}$ by the 
$\mathrm{V}(\mathrm{t})$ pulse, bringing the junction from HRS to LRS. In the second $\mathrm{V}(\mathrm{t})$ excursion, after zeroing $\mathrm{V}$, the I-V curve (red curve) is radically different. As seen, the sample now reversibly follows the trace of the LRS state, thus indicating that it remains in the LRS and signifying that, in absence of a poling V $>0$ field, $\mathrm{P}^{\downarrow}$ domains have not switched back to the HRS $\left(\mathrm{P}^{\uparrow}\right)$. This observation implies that the imprint field revealed by data Figure 3 does not have a significant role while setting the LRS, because $\mathrm{E}_{\text {imp }}$ favors LRS. As it could be argued that the backswitching process could be a slow process and thus not perceptible in the time scale of the measurement, we have performed similar measurements but using a longer dwell time $\tau_{D}=1 \mathrm{~s}$ at $\mathrm{V}=0$. The results (Figure $5 \mathrm{~b}$ ) display a similar behavior than in Figure 5a, thus confirming that backswitching from LRS to HRS is not relevant.
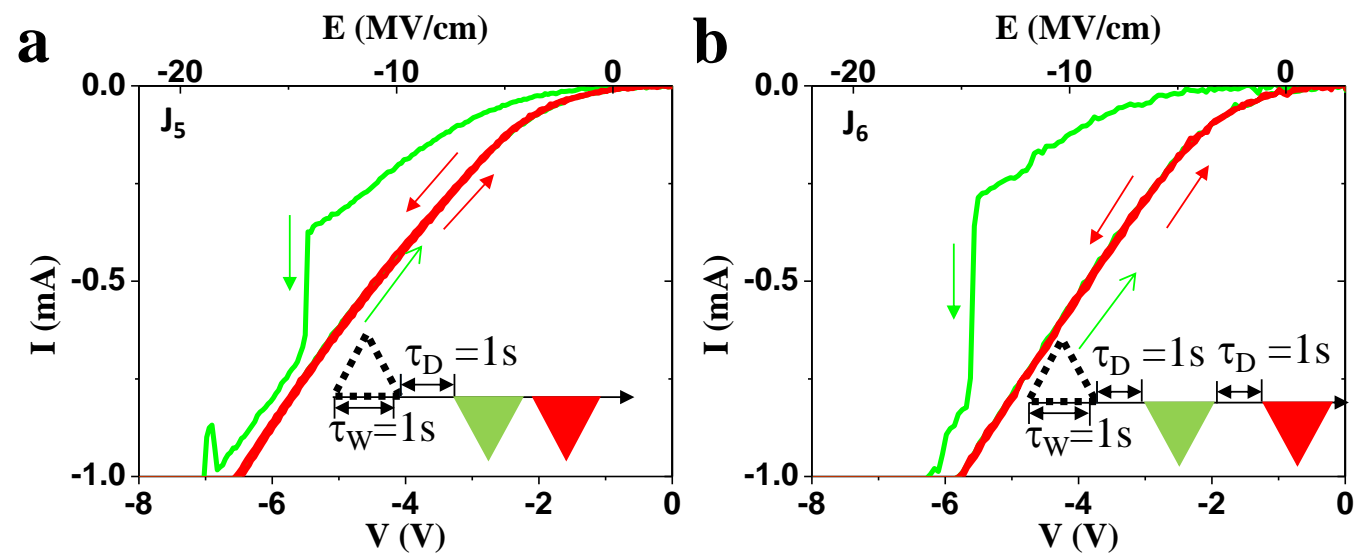

Figure 5. I-V reading pulses after suitable prepoling. Prior to measurements, the junctions have been pre-poled with a $\mathrm{V}=+8 \mathrm{~V}$ triangular pulse of $\tau_{W}=1 \mathrm{~s}$. Sketches indicate the sequence of prepoling writing (dashed triangle) and reading voltages $\mathrm{V}(\mathrm{t})$ pulses (green and read triangles). Reading I-V curves collected at $10 \mathrm{~Hz}$ with no delay $\left(\tau_{D}=0 \mathrm{~s}\right.$ ) between writing and reading (a), and with a delay $\left(\tau_{D}=1 \mathrm{~s}\right)$ between consecutive reading pulses (b).

We turn now to the tunneling resistance variation upon electric poling of these junctions. Measurements were performed by writing a polar state using a triangular $\mathrm{V}(\mathrm{t})$ pulse of varying amplitude up to $\pm 8 \mathrm{~V}$, of duration $\tau_{w}$. Afterwards, the junction resistance is measured at $100 \mathrm{mV}$. In Figure 6a, we show data written at $\tau_{w}=20 \mu$ s. It can be appreciated that after a $\mathrm{V}>+8 \mathrm{~V}$ pulse (electric field pointing down from Pt to LSMO) a HRS is written. It remains stable until $\mathrm{V}_{\mathrm{C}-} \approx-7 \mathrm{~V}$ where a sudden switch to the LRS is observed. The electroresistance (TER) is quantified by: TER = $[\mathrm{R}(\mathrm{HRS})-\mathrm{R}(\mathrm{LRS})] / \mathrm{R}(\mathrm{LRS})$ where R(HRS,LRS) are the junction resistance in HRS or LRS states, 
respectively. It can be derived from data in Figure 6 a that TER $\approx 230 \%$. This is a remarkably large TER value for micrometric sized $\left(250 \mu \mathrm{m}^{2}\right)$ metal/BTO/metal junction at room-temperature. ${ }^{[14-17]}$ In the reverse side of the loop, switching from LRS to a HRS occurs at a somewhat relatively smaller

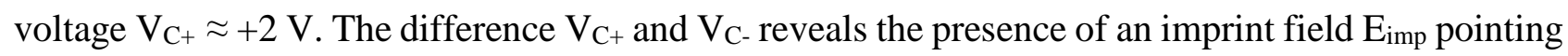
downwards (from Pt towards LSMO) in agreement with data in Figure 3. In Figure 6c, we plot the I$\mathrm{V}$ curve measured in the $\pm 8 \mathrm{~V}$ range at using a $25 \mathrm{kHz} \mathrm{V}(\mathrm{t})$ excursion which correspond to a writing time $\tau_{w}$ of $20 \mu \mathrm{s}$, identical to that used $\left(\tau_{w}=20 \mu \mathrm{s}\right)$ in Figure 6a. There is an obvious coincidence between the switching peaks observed in I-V curves (Figure 6c) and the electroresistance changes (Figure 6a). When longer writing pulses are used, for instance $\tau_{w}=0.5 \mathrm{~s}$ in Figure $6 \mathrm{~b}$, after setting the HRS with a $\mathrm{V}=+8 \mathrm{~V}$ pulse, the transition to the LRS when reducing writing voltage, starts gradually already at $\mathrm{V} \approx 0$ and the complete transition is definitely more gradual than that observed using shorter writing pulses (Figure $6 \mathrm{a}, \tau_{w}=20 \mu \mathrm{s}$ ). The corresponding I-V curves are shown in Figure $6 \mathrm{c}, \mathrm{d}$. They have been recorded at $1 \mathrm{~Hz}$ (corresponding to $\tau_{w}=0.5 \mathrm{~s}$ of Figure $6 \mathrm{c}$ ). The close coincidence between switching peaks and TER changes is also well apparent.

It can be suspected that differences on polarization switching should be more apparent at low driving fields, namely at subcoercive bias fields $\left(\mathrm{V}<\left|\mathrm{V}_{\mathrm{C}+,-}\right|\right)$. Therefore, we explore the time stability the HRS and LRS states under subcoercive $(\mathrm{V}>0$ and $\mathrm{V}<0)$ bias, once written by suitable voltages. We first write a $\mathrm{P}^{\uparrow}$ state by applying $\mathrm{V}=-8 \mathrm{~V}\left(\tau_{w}=1 \mathrm{~s}\right)$ pulse to set the LRS. We subsequently apply a positive reading voltage $\mathrm{V}_{\mathrm{r}}=+2 \mathrm{~V}$, as sketched in Figure $7 \mathrm{a}$. Notice that $\mathrm{V}<\mathrm{V}_{\mathrm{C}+}$, thus the set voltage is slightly smaller than the voltage where the switch peak in $\mathrm{I}(\mathrm{V}>0)$ occurs. The results, displayed in Figure $7 b$, indicate that, in spite that $\mathrm{V}_{\mathrm{r}}<\mathrm{V}_{\mathrm{C}+}$, a gradual evolution with time from the LRS to HRS occurs. The I $(\mathrm{t})$ time-dependence of Figure $7 \mathrm{~b}$ can be well describe by an stretched exponential behavior (dashed line) (See Supplementary Information S5) as expected from the KolmogorovAvrami-Ishibashi (KAI) model ${ }^{[21,39]}$ suggesting that a random nucleation and lateral expansion until coalescence of $\mathrm{P}^{\downarrow}$ domains. Similar conclusion had been reported earlier in $\mathrm{BaTiO}_{3}$ thin films, ${ }^{[30]}$ However, numerical analysis of the KAI fit (Supplementary Information S5) shows that the extracted 
exponent in the stretched exponential is smaller $(\mathrm{n} \approx 0.64)$ than the smallest possible value $(\mathrm{n}=1)$ (bounded by the dimensionality (D> 1) of the domain growth system). This indicate that the simplifying assumptions of the KIA model are not fulfilled. Similar discrepancy has been often encountered in ferroelectric thin films. Jo et al. ${ }^{[22]}$ suggested that a Lorentzian distribution of nucleation times, associated to local field variation originating from defect dipoles or others at domain pinning sites, could account for the observed $\mathrm{n}<1$. In the present case, the unavoidable surface and interface roughness could contribute to the suggested distribution of nucleation times.
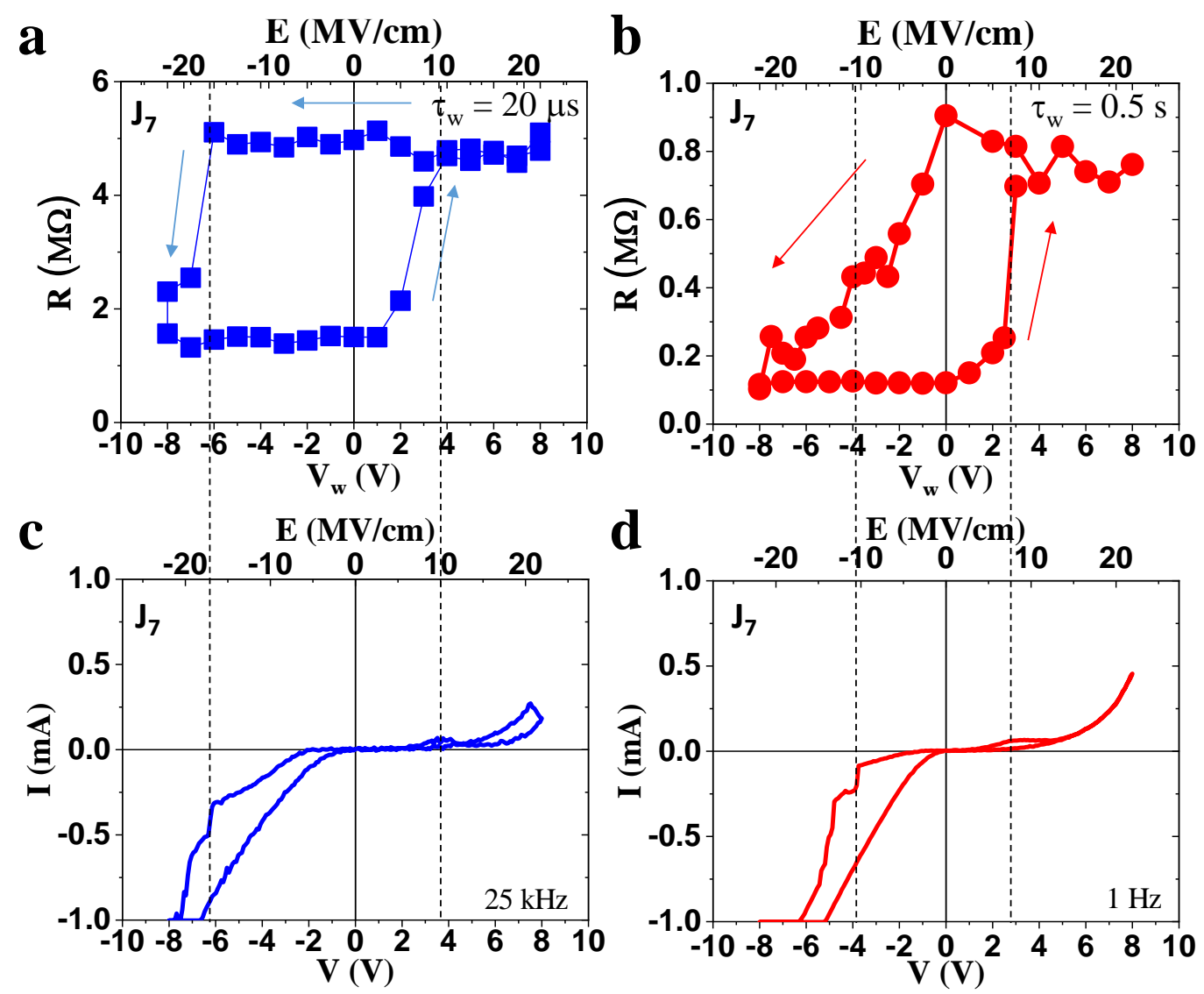

Figure 6. Electroresistance recorded in a Pt/BTO/LSMO $\left(\mathrm{J}_{7}\right)$ junction after writing pulses (up to $\pm 8 \mathrm{~V}$ ) of duration: (a) $\tau_{w}=20 \mu \mathrm{s}$ and (b) $\tau_{w}=0.5 \mathrm{~s}$. I-V curves recorded using identical writing times as in (a,b): (c) $\tau_{w}=20 \mu \mathrm{s}$ and (d) $\tau_{w}=0.5 \mathrm{~s}$.

Next, we write $\mathrm{P}^{\downarrow}$ state by applying a $\mathrm{V}=+8 \mathrm{~V}\left(\tau_{\mathrm{w}}=1 \mathrm{~s}\right)$. We subsequently apply a negative reading voltage $V_{r}=-4.5 \mathrm{~V}$. The measuring sequence is sketched in Figure $7 \mathrm{~d}$, and we record the time evolution of the charge current (proportional to the junction resistance). Results are shown in Figure 7e. Notice that the $V_{r}$ has a magnitude smaller than the corresponding coercive voltage $\left(V_{C}\right)\left(\mid V_{r}\right.$ 
$\left.|<| V_{C-} \mid\right)$. In spite of this, it is obvious in Figure 7e that the sample displays sudden changes of resistance at some specific instants, that are analogous to the avalanches observed when increasing $\mathrm{V}$ $(<0)$ (as in Figure 1a, 1b and 4a-d). These data illustrate the clear differences of the polar domain switching dynamics under $\mathrm{V}>0$ and $\mathrm{V}<0$ bias.
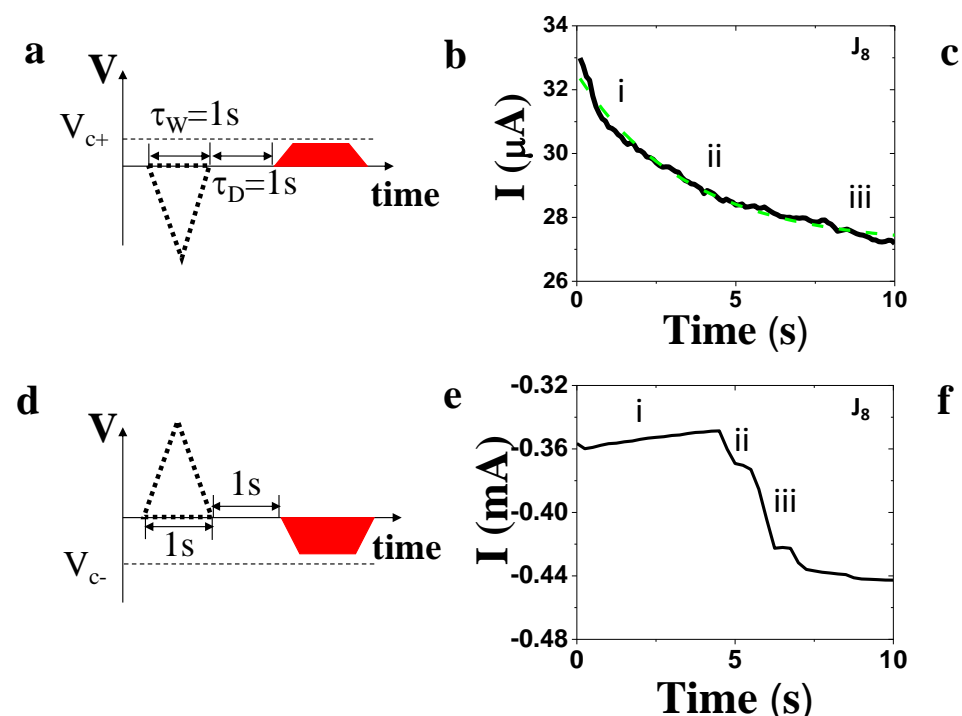

c
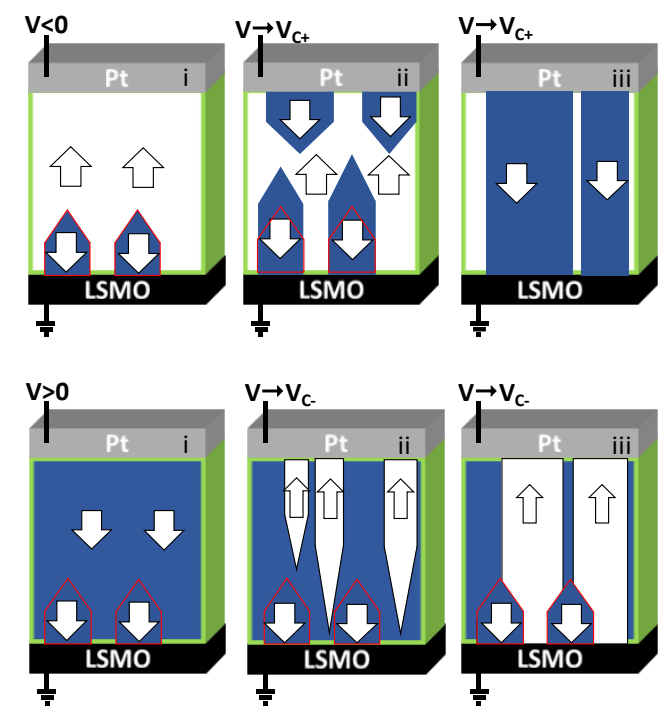

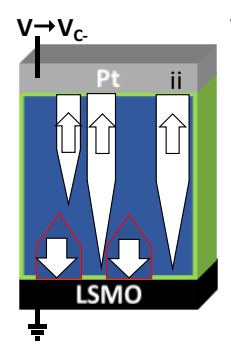

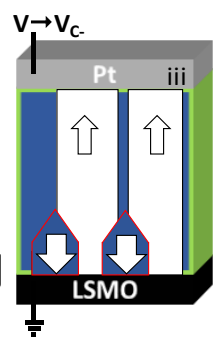

Figure 7. (a) Sketch of measurement sequence for $0<\mathrm{V}<\mathrm{V}_{\mathrm{C}+}$; Notice that the reading $\mathrm{V}$ voltage (red trapezoid) is smaller than the coercive field $\mathrm{V}_{\mathrm{C}+}$ (dashed line). (b) Time-dependent current $\mathrm{I}(\mathrm{t})$ collected at constant reading $\mathrm{V}$ voltage $\left(0<\mathrm{V}<\mathrm{V}_{\mathrm{C}_{+}}\right)$(solid line). Dashed line indicates the result of the data fit using a stretched exponential decay (see text). (c) Sketch indicating of gradual growth of unswitchable $\mathrm{P}^{\downarrow}$ domains (outlined in red) assisted by the pointing down (towards LSMO) imprint field (i,ii,iii). (d) Sketch of measurement sequence for $\mathrm{V}_{\mathrm{C}-}<\mathrm{V}<0$. (e) Time-dependent current $\mathrm{I}(\mathrm{t})$ collected at constant reading $\mathrm{V}\left(\mathrm{V}_{\mathrm{C}-}<\mathrm{V}<0\right)$. (f) Sketch indicating of the abrupt domain switching when the bias field acts against unswitchable polar domains (outlined in red) and imprint; domains of different sizes or bundles of domains switch forming $\mathrm{P}^{\uparrow}$ domain avalanches (i,ii,iii).

Based on all data above, we devise a simple microstructural model than accounts for the observed differences. In Figures $7 \mathrm{c}$ and $7 \mathrm{f}$ we sketch the ferroelectric domain evolution as a function of the external bias as derived from the experimental data, and explicitly considering: the existence of an imprint field ( $\mathrm{E}_{\mathrm{imp}}$ ) pointing down (from Pt to LSMO) and the different dynamics of HRS to LRS and LRS to HRS processes. The imprint field in ferroelectric thin films may have a variety of origins, including the presence of unswitchable domains, asymmetric electrostatic boundary conditions, or the presence of polar defects (oxygen vacancies for instance) just to mention a few. For simplicity in the sketches in Figures $7(\mathrm{c}, \mathrm{f})$, we assume here that imprint is associated to the presence of unswitchable polar domains and as a sake of sketch clarity, we locate them at the LSMO/BTO 
interface (outlined in red). We describe first the results of Figure 7b, starting from an initially poledup $\left(\mathrm{P}^{\uparrow}\right)$ state $[(\mathrm{i})$ in Figure $7 \mathrm{c}]$ obtained by a suitable $\mathrm{V}<0$ pulse. When a voltage $\mathrm{V}>0\left(\mathrm{~V}<\mathrm{V}_{\mathrm{C}+}\right)$ is applied (as in Figure 7a,b), the pre-existing $\mathrm{P}^{\downarrow}$ and new $\mathrm{P}^{\downarrow}$ domains [(ii) and (iii) in Figure 7c]. Thus along the i-ii-iii path the junction resistance gradually increases (because $\mathrm{P}^{\downarrow}$ corresponds to HRS) in agreement with the experimental observation [Figure 7b]. This nucleation and expansion of $\mathrm{P}^{\downarrow}$ is the process that can be roughly described by a modified KIA process as described above. We next describe the reverse switching process from $\mathrm{P}^{\downarrow}$ to $\mathrm{P}^{\uparrow}$ under a subcoercive voltage bias (experimental data Figure 7e). We start from a $\mathrm{P}^{\downarrow}$ state [(i) in Figure 7f] obtained by application of a suitable V $>0$ pulse and zeroing it (Figure 7d). We subsequently apply a negative $\mathrm{V}<0$ field, smaller that the corresponding coercive field ( $|\mathrm{V}|<\left|\mathrm{V}_{\mathrm{C}-}\right|$, Figure $\left.7 \mathrm{~d}\right)$ and we follow the evolution with time of the junction resistance. Under negative bias, $\mathrm{P}^{\uparrow}$ domains should nucleate and expand. We notice however that the presence of pinned $\mathrm{P}^{\downarrow}$ domains should locally stabilize the $\mathrm{P}^{\downarrow}$ domains due to the energyfavored tail-to-head relative ordering. Moreover, the nucleation process of reverse $\left(\mathrm{P}^{\downarrow}\right)$ domains should be modified by the presence of the imprint field. Indeed, the critical radius of domain nucleation is proportional to $\sim 1 /\left(\mathrm{V} \pm \mathrm{z} \cdot \mathrm{E}_{\mathrm{imp}}\right)$ where $\mathrm{V}$ is the potential applied and $\left(\mathrm{z} \cdot \mathrm{E}_{\mathrm{imp}}\right)$ is a descriptor of the local voltage associated to the imprint field, and + (-) sign apply when the imprint field adds (opposes) to the applied field. ${ }^{[27,28]}$ Therefore, the critical radius gets larger when the applied field $\mathrm{E}$ is antiparallel to $\mathrm{E}_{\mathrm{imp}}$ thus retarding nucleation, enhancing domain wall pinning and ultimately hampering domain motion. Upon increasing applied voltage, domains walls eventually depin and bundles of domain move a certain distance to a new pining site, in avalanche-like process, as observed in PZT thin films [24,34]. In short, bundles of $\mathrm{P}^{\downarrow}$ domains switch to $\mathrm{P}^{\uparrow}$, as depicted in steps [(ii), (iii) in Figure 7f] and correspondingly, jumps of the increasing conductance are observed in the $\mathrm{I}(\mathrm{t})$ data (Figure $7 \mathrm{e})$. 


\section{Discussion and Conclusion}

The experiments above show that asymmetric resistive switching dynamics observed in BTO tunnel devices, can be directly linked to the presence of electric field imprint likely associated, but not necessarily, to the presence of unswitchable polar regions. They induce asymmetric domain grow and switching depending on the polarity of the driving filed with respect to the unswitchable domains. Here, we have observed and analyzed the asymmetry of the switching process on the basis of the electroresistance (I-V) data of nanometric thin tunnel junctions. Recently Balke et al. obtained a direct visualization by piezo force microscopy of an asymmetric switching process in symmetric parallel planar $\mathrm{SrRuO}_{3} / \mathrm{BiFeO}_{3} / \mathrm{SrRuO}_{3}$ capacitors ${ }^{[29]}$. In agreement with current theories ${ }^{[25,40,41]}$ and earlier experiments $^{[30]}$ of preferential nucleation in one electrode in symmetric capacitors, they also conclude that a build-in electric field at the ferroelectric/electrode breaks the symmetry of the capacitor geometry and ultimately leads to different switching behavior for positive or negative switching voltages.

Asymmetric resistive switching has been observed in other resistive switching devices such as in valence change memories (VCM), and more precisely in filamentary-type VCM. These devices typically display a strong asymmetry in the HRS to LRS switching process. It has been recently demonstrated that this asymmetric switching can be directly related to an asymmetric dissipation than in some cases can also stimulate and control ionic motion, with concurrent impact on device resistance. ${ }^{[42]}$ Our data indicate that avalanches are visible when the system switches from HRS-toLRS but not in the LRS-to-HRS switching, and thus one may wonder if domain avalanches are assisted by the higher power dissipated at the junctions when in the HRS. The observation (Figures 4a-d) that when increasing frequency (that is: shorter $\mathrm{V}(\mathrm{t})$ and thus less power dissipates at the device when in the HRS) the switching occurs at higher $V_{c-}$ voltage, appears at first sight to be also compatible with a possible occurrence of thermal effects assisting or promoting domain avalanches. The present data at first sight, do not univocally exclude any of the suggested mechanisms. However, the experimental observation that the power consumption of the Pt/BTO/LSMO junctions in the HRS- 
to-LRS transition at $\mathrm{V}<0$, where avalanches occurs, is smaller $(30 \%)$ than the one consumed at the LRS-to-HRS switching peak (V > 0) (see Fig.S6, in Supplementary Information) indicates that thermal effects are not the driving force for the assymetric switching observed in micrometric (this work, see also in Ref [43] and nanometric [17] $\mathrm{BaTiO}_{3}$ tunnel barriers.

In the scenarios above, obtaining symmetric switching dynamics for positive and negatives seems to be intrinsically very challenging. However, the observed asymmetry can be properly handled; for instance, in ferroelectric memories, unipolar reading of the memory state (from LRS to HRS) appears to lead to steady and provide a robust output; negative reading could also be handled if the abrupt conductance jumps are nor detrimental to digital data storage. However, the results presented here may have more important implications for the integration of ferroelectric materials in neuromorphic computing, where knowing time-dependent evolution of the potentials triggering the presynapse responses is crucial and, as argued here, this is much depending on electrostatic and microstructural boundary conditions at the electrode/ferroelectric layer.

\section{Experimental Section}

Samples growth and geometry. Nanometric ferroelectric capacitors have been fabricated by pulsed laser deposition by growing thin films of ferroelectric $(3.6 \mathrm{~nm}) \mathrm{BaTiO}_{3}(\mathrm{BTO})$ on top of an epitaxial metallic $\mathrm{La}_{0.67} \mathrm{Sr}_{0.33} \mathrm{MnO}_{3}$ (LSMO) (30 nm) layer deposited on $\mathrm{SrTiO}_{3}(001)$ substrates. Details of growth conditions and structural characterization of the films have been reported elsewhere. ${ }^{[16,44]}$ Circular Pt electrodes (20 nm thick, area $250 \mu \mathrm{m}^{2}$ ) were subsequently deposited ex-situ, by sputtering, on the BTO film surface by using a shadow mask. About 300 electrodes, labelled $\mathrm{J}_{\mathrm{N}}$, where $\mathrm{N}$ is the reference number for each electrode, are prepared simultaneously.

Electric characterization. Current-voltage I-V curves have been obtained by applying triangular V(t) pulses of different amplitude $\mathrm{V}$ and frequency $(v)$, using the contact configuration sketched in Figure 1b. The polarization state of the junctions was set (written) by applying a triangular pulse of a given amplitude and duration $\left(\mathrm{V}, \tau_{\mathrm{w}}\right)$. In all cases the bottom electrode is grounded and the $\mathrm{V}(\mathrm{t})$ signal is 
applied to the top electrode $(\mathrm{Pt})$. Measurements have been done at room temperature using an AixACCT TFAnalyser2000 platform.

\section{Acknowledgments}

Financial support from the Spanish Government, through the "Severo Ochoa" Programme for Centres of Excellence in R\&D (SEV-2015-0496) and the MAT2017-85232-R, MAT2014-56063-C2-1-R, and MAT2015-73839-JIN projects, and from Generalitat de Catalunya (2017 SGR 1377) is acknowledged. MQ is financially supported by China Scholarship Council (CSC) with No. 201406890019. MQ work has been done as a part of her Ph.D. program in Materials Science at Universitat Autònoma de Barcelona.

\section{References}

[1] V. Garcia, M. Bibes, Nat. Commun. 2014, 5, 4289.

[2] E. Y. Tsymbal, H. Kohlstedt, Science 2006, 313, 181.

[3] E. Y. Tsymbal, A. Gruverman, V. Garcia, M. Bibes, A. Barthélémy, MRS Bull. 2012, 37, 138.

[4] Z. Wen, C. Li, D. Wu, A. Li, N. Ming, Nat. Mater. 2013, 12, 617.

[5] H. Yamada, V. Garcia, S. Fusil, S. Boyn, M. Marinova, A. Gloter, S. Xavier, J. Grollier, E. Jacquet, C. Carrétéro, C. Deranlot, M. Bibes, A. Barthélémy, ACS Nano 2013, 7, 5385.

[6] D. Pantel, S. Goetze, D. Hesse, M. Alexe, Nat. Mater. 2012, 11, 289.

[7] D. Pantel, H. Lu, S. Goetze, P. Werner, D. Jik Kim, A. Gruverman, D. Hesse, M. Alexe, Appl. Phys. Lett. 2012, 100, 232902.

[8] X. S. Gao, J. M. Liu, K. Au, J. Y. Dai, Appl. Phys. Lett. 2012, 101, 142905.

[9] Y. W. Yin, M. Raju, W. J. Hu, X. J. Weng, K. Zou, J. Zhu, X. G. Li, Z. D. Zhang, Q. Li, Front. Phys. 2012, 7, 380.

[10] A. Zenkevich, Y. Matveyev, K. Maksimova, R. Gaynutdinov, A. Tolstikhina, V. Fridkin, Phys. Rev. B 2014, 90, 161409.

[11] S. Boyn, S. Girod, V. Garcia, S. Fusil, S. Xavier, C. Deranlot, H. Yamada, C. Carrétéro, E. Jacquet, M. Bibes, A. Barthélémy, J. Grollier, Appl. Phys. Lett. 2014, 104, 052909.

[12] A. Gruverman, D. Wu, H. Lu, Y. Wang, H. W. Jang, C. M. Folkman, M. Y. Zhuravlev, D. Felker, M. Rzchowski, C.-B. Eom, E. Y. Tsymbal, Nano Lett. 2009, 9, 3539.

[13] L. Wang, M. R. Cho, Y. J. Shin, J. R. Kim, S. Das, J. G. Yoon, J. S. Chung, T. W. Noh, Nano Lett. 2016, 16, 3911.

[14] D. J. Kim, H. Lu, S. Ryu, C. W. Bark, C. B. Eom, E. Y. Tsymbal, a. Gruverman, Nano Lett. 2012, 12, 5697.

[15] A. Chanthbouala, A. Crassous, V. Garcia, K. Bouzehouane, S. Fusil, X. Moya, J. Allibe, B. Dlubak, J. Grollier, S. Xavier, C. Deranlot, A. Moshar, R. Proksch, N. D. Mathur, M. Bibes, A. Barthélémy, Nat. Nanotechnol. 2012, 7, 101.

[16] G. Radaelli, D. Gutiérrez, F. Sánchez, R. Bertacco, M. Stengel, J. Fontcuberta, Adv. Mater. 2015, 27, 2602.

[17] A. Chanthbouala, V. Garcia, R. O. Cherifi, K. Bouzehouane, S. Fusil, X. Moya, S. Xavier, H. Yamada, C. Deranlot, N. D. Mathur, M. Bibes, A. Barthélémy, J. Grollier, Nat. Mater. 2012, 11, 860.

[18] C. S. Hwang, Adv. Electron. Mater. 2015, 1, 1400056.

[19] W. J. Merz, Phys. Rev. 1954, 95, 690.

[20] Y. H. Shin, I. Grinberg, I. W. Chen, A. M. Rappe, Nature 2007, 449, 881.

[21] Y. Ishibashi, Y. Takagi, J. Phys. Soc. Japan 1971, 31, 506.

[22] J. Y. Jo, H. S. Han, J. G. Yoon, T. K. Song, S. H. Kim, T. W. Noh, Phys. Rev. Lett. 2007, 99, 267602.

[23] A. K. Tagantsev, G. Gerra, J. Appl. Phys. 2006, 100, 051607.

[24] G. Gerra, A. K. Tagantsev, N. Setter, Phys. Rev. Lett. 2007, 98, 207601. 
[25] G. Gerra, A. K. Tagantsev, N. Setter, Phys. Rev. Lett. 2005, 94, 107602.

[26] S. M. Yang, J. G. Yoon, T. W. Noh, Curr. Appl. Phys. 2011, 11, 1111.

[27] S. Jesse, B. J. Rodriguez, S. Choudhury, A. P. Baddorf, I. Vrejoiu, D. Hesse, M. Alexe, E. A. Eliseev, A. N. Morozovska, J. Zhang, L. Q. Chen, S. V. Kalinin, Nat. Mater. 2008, 7, 209.

[28] P. Maksymovych, N. Balke, S. Jesse, M. Huijben, R. Ramesh, A. P. Baddorf, S. V. Kalinin, J. Mater. Sci. 2009, 44, 5095.

[29] N. Balke, M. Gajek, A. K. Tagantsev, L. W. Martin, Y. H. Chu, R. Ramesh, S. V. Kalinin, Adv. Funct. Mater. 2010, 20, 3466.

[30] J. Li, Z. Liu, B. W. Wessels, J. Appl. Phys. 2010, 107, 124106.

[31] M. Ignatov, M. Ziegler, M. Hansen, H. Kohlstedt, Sci. Adv. 2017, 3, e1700849.

[32] J. F. Scott, Integr. Ferroelectr. 1996, 12, 71.

[33] Z. Liu, A. L. Meier, B. W. Wessels, J. Appl. Phys. 2008, 104, 064115.

[34] R. J. Harrison, E. K. H. Salje, Appl. Phys. Lett. 2010, 97, 021907.

[35] E. K. H. Salje, X. Wang, X. Ding, J. F. Scott, Adv. Funct. Mater. 2017, 27, 1700367.

[36] D. J. Kim, J. Y. Jo, Y. S. Kim, T. K. Song, J. Phys. D. Appl. Phys. 2010, 43, 395403.

[37] P. Bintachitt, S. Jesse, D. Damjanovic, Y. Han, I. M. Reaney, S. Trolier-McKinstry, S. V. Kalinin, Proc. Natl. Acad. Sci. 2010, 107, 7219.

[38] A. Planes, E. Vives, J. Phys. Condens. Matter 2017, 29, 334001.

[39] M. Avrami, J. Chem. Phys. 1940, 8, 212.

[40] A. K. Tagantsev, C. Z. Pawlaczyk, K. Brooks, N. Setter, Integr. Ferroelectr. 1994, 4, 1.

[41] C. J. Brennan, Integr. Ferroelectr. 1995, 7, 93.

[42] M. von Witzleben, K. Fleck, C. Funck, B. Baumkötter, M. Zuric, A. Idt, T. Breuer, R. Waser, U. Böttger, S. Menzel, Adv. Electron. Mater. 2017, 3, 1700294.

[43] Z. Xi, C. Zheng, Z. Wen, ACS Appl. Mater. Interfaces 2018, 10, 6024.

[44] G. Radaelli, D. Gutiérrez, M. Qian, I. Fina, F. Sánchez, L. Baldrati, J. Heidler, C. Piamonteze, R. Bertacco, J. Fontcuberta, Adv. Electron. Mater. 2016, 1600368. 
Asymmetric resistive switching dynamics in ferroelectric tunnel junctions.

Deterministic and smooth transitions are observed from low-to-high resistance states but avalanchelike behavior is observed in the reverse process. An imprint field breaks the symmetry of the junction and determines either a gradual nucleation and expansion of ferroelectric domains or sudden domain-bundles switching and concomitantly, different resistive switching dynamics depending on the biasing electric field.

Keywords: ferroelectric tunnel junctions, polarization switching dynamics, $\mathrm{BaTiO}_{3}$ films, memristor, neuromorphic computing

Mengdi Qian, Ignasi Fina,* Florencio Sánchez and Josep Fontcuberta*

Asymmetric Resistive Switching Dynamics in BaTiOz Tunnel Junctions

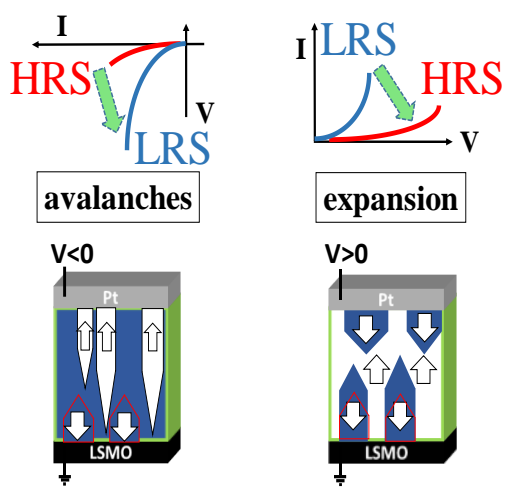




\section{Supporting Information}

\section{Asymmetric Resistive Switching Dynamics in BaTiOz Tunnel Junctions}

Mengdi Qian, Ignasi Fina, * Florencio Sánchez and Josep Fontcuberta*

(a)

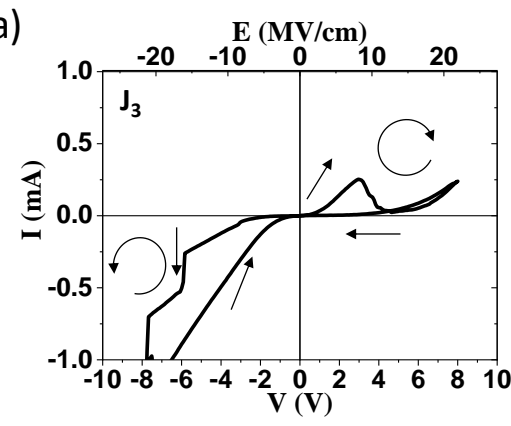

(b)

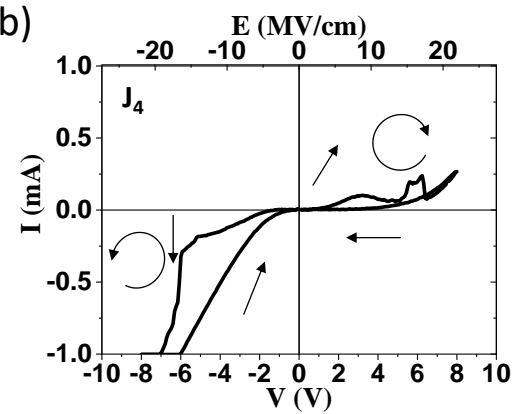

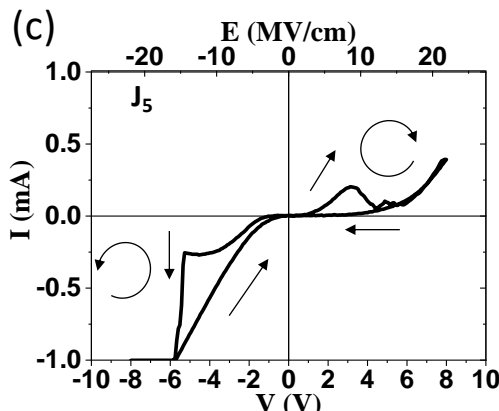

Figure S1. The I-V loops following the $-8 \mathrm{~V}$ to $+8 \mathrm{~V}$ to $-8 \mathrm{~V}$ path of three different junctions $\mathrm{J}_{3}$ (a), $\mathrm{J}_{4}(\mathrm{~b})$ and $\mathrm{J}_{5}(\mathrm{c})$.

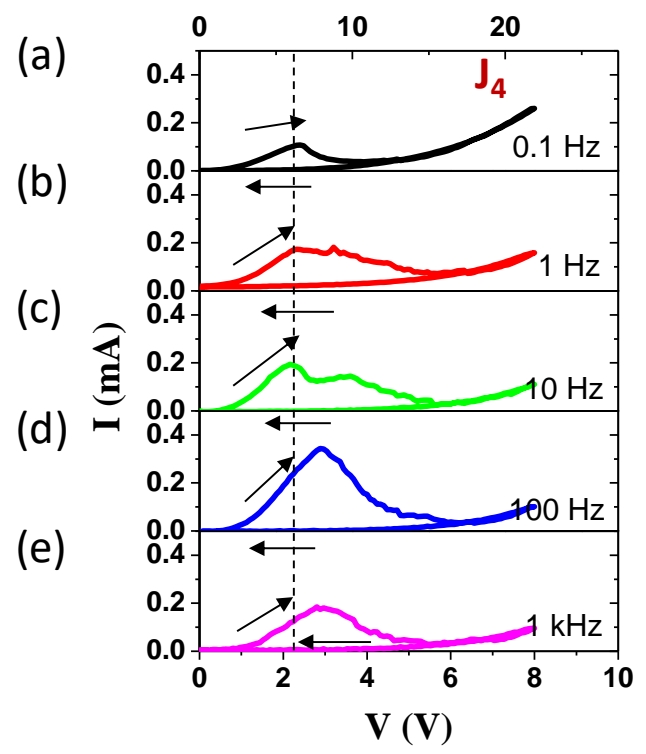

Figure S2. Positive V $>0$ branch of the $\mathrm{I}-\mathrm{V}$ loops of junction $\mathrm{J}_{4}$ recorded using triangular pulses of $0.1 \mathrm{~Hz}$ (a), $1 \mathrm{~Hz}$ (b), $10 \mathrm{~Hz}$ (c), $100 \mathrm{~Hz}$ (d) and $1 \mathrm{kHz}$ (e). Prior to measurement the junction have been pre-poled with a $\mathrm{V}=-8 \mathrm{~V}$ triangular pulse of $\tau_{W}=1 \mathrm{~s}$. 

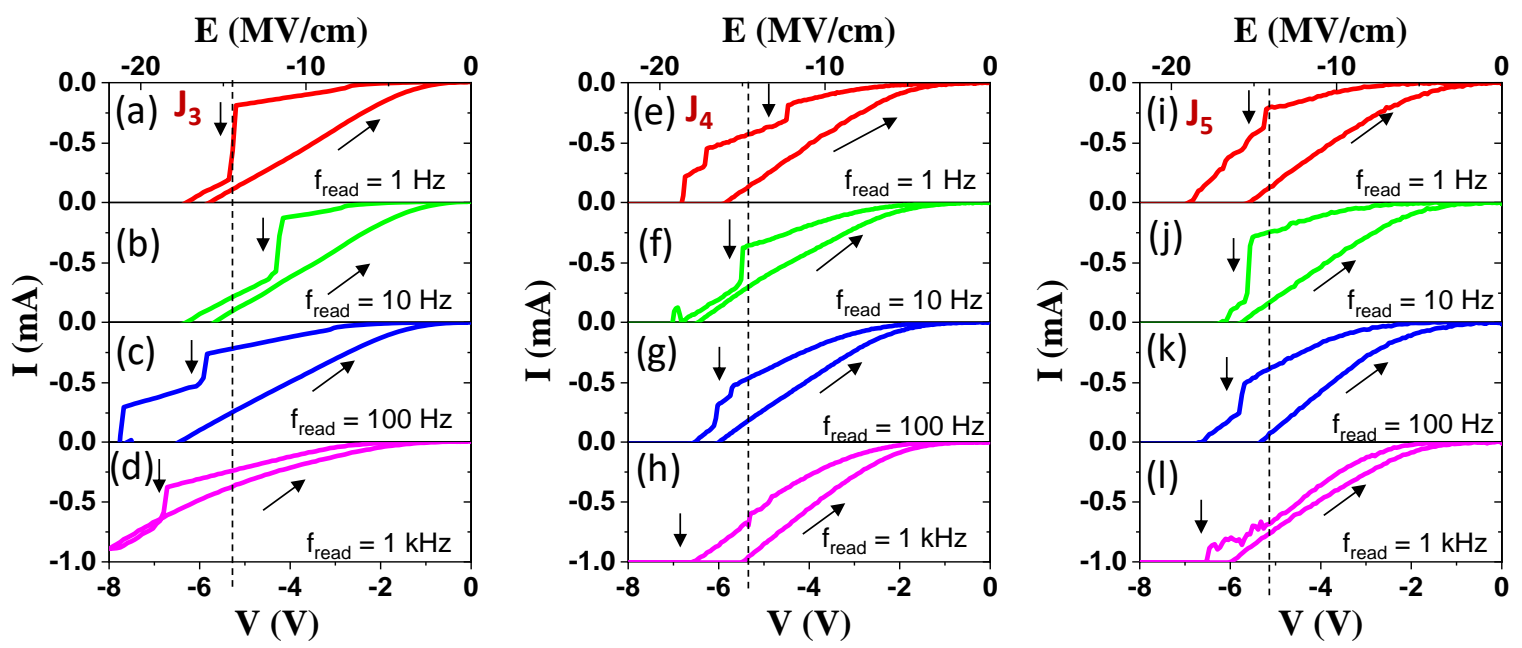

Figure S3. Dependence on the measuring (reading) frequency of the HRS to LRS transitions in the reverse side $(\mathrm{V}<0)$. Prior to measurement the junctions have been pre-poled with a $\mathrm{V}=+8 \mathrm{~V}$ triangular pulse of $\tau_{W}=1 \mathrm{~s} . \mathrm{J}_{3}(\mathrm{a}-\mathrm{d}), \mathrm{J}_{4}(\mathrm{e}-\mathrm{h})$ and $\mathrm{J}_{5}(\mathrm{i}-\mathrm{l})$.

(a)

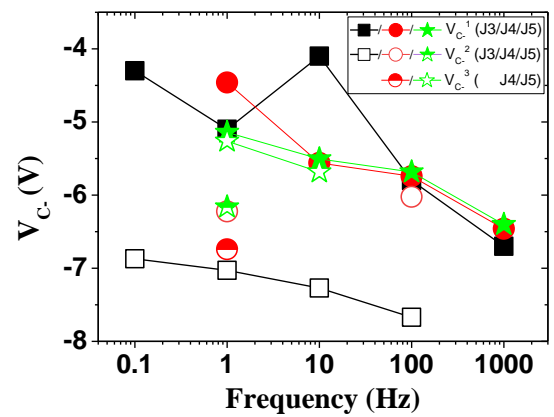

(b)

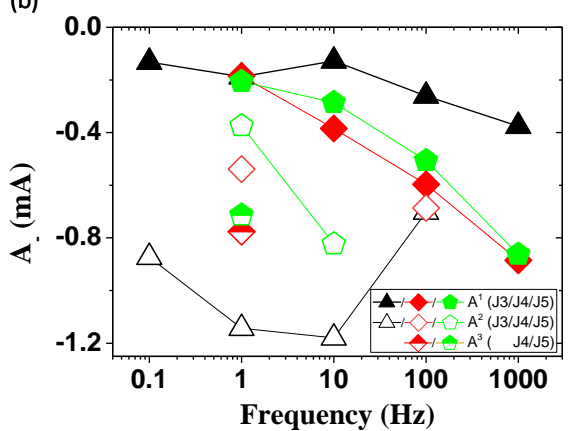

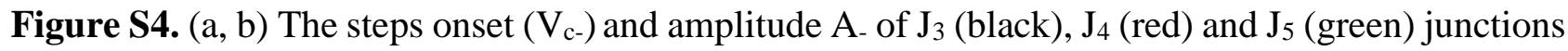
versus of frequency.
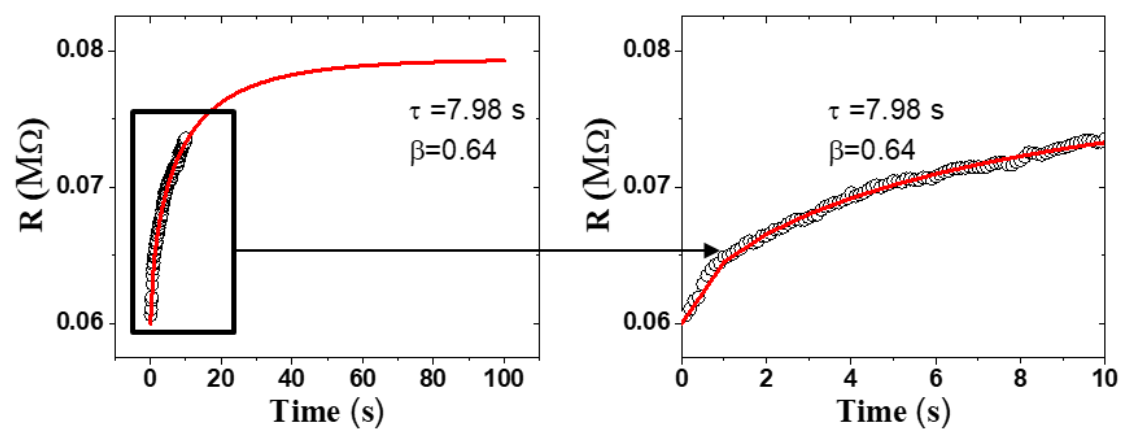

Figure S5. Time-dependente $\mathrm{I}(\mathrm{t})$ data collecte in the Pt/BTO/LSMO junction $\left(\mathrm{J}_{8}\right)$ after setting the LRS by an appropriate $\mathrm{V}<0$ wriring pulse (sketch of measuring sequence in Figure 7a) Data collected (open symbols) and fitted data (red curves) using $\mathrm{r}(\mathrm{t})=r 1+r 2\left\{1-\exp \left[-\left(\frac{t}{\tau}\right)^{\beta}\right]\right\}$. Fitting parameters are indicated. 


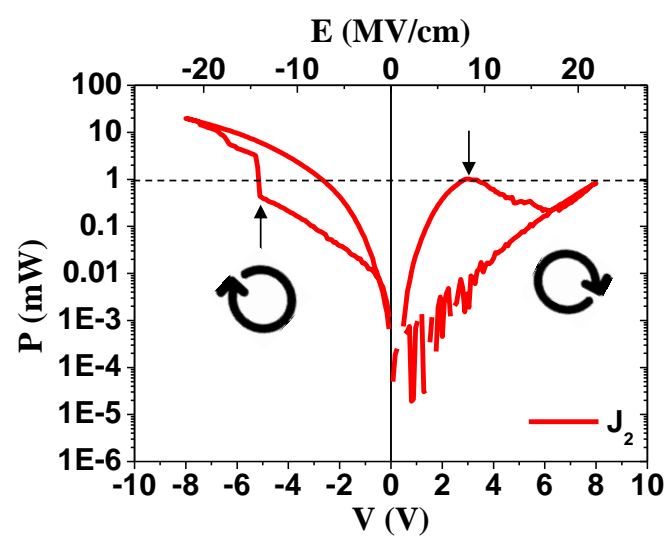

Figure S6. Power consumption $\mathrm{P}$ of the Pt/BTO/LSMO junction $\left(\mathrm{J}_{2}\right)$. The correspondinh IV curve is shown in Figure 1c. It can be appreciated that avalanche switching visible in the $\mathrm{V}<0$ section of the I-V curve (pointing-up arrow), which correspond to the HRS to LRS transition, occurs at lover power (30\%) than the one consumed at the LRS to HRS switching peak observed in the $\mathrm{V}>0$ region (pointing-down arrow). This observation suggest that thermal dissipation effects are not governing the asymetry of HRs-to-LRS trabsitions and viceversa. 